\title{
Riscos e benefícios da suplementação medicamentosa de cálcio nos idosos
}

\section{Risks and benefits of calcium drug supplementation in elderly}

Nadielle Silva BIDU

Faculdade Independente do Nordeste - FAINOR. Av. Luiz Eduardo Magalhães, 1035 Candeias, Vitória da Conquista - BA, 45055-420,Brasil.E-mail: nadiellebidu@gmail.com

\begin{abstract}
Calcium is a mineral of unique importance in the human body, exerting important cellular functions, mainly on the skeletal system. With aging, some organs undergo changes that compromise its operation, as in the case of bone which has its density reduced from aging, increased risk of fractures and bone diseases such as, for example, osteoporosis. One option to reduce this wear bone is the use of medications based on calcium salts, which have different concentrations of calcium supplements, in addition to requiring a great stomach $\mathrm{pH}$ for absorption and have different degrees of solubility and can therefore be used in different clinical situations. Besides the reduction of bone loss, such treatment has other benefits such as lowering blood pressure and reducing dyslipidemia, but is also associated with vascular calcification and other risks that increases mortality in patients, and promote the accumulation of heavy metals. As these supplements can be purchased without a prescription, they are easily within reach of the elderly population that is induced by the advertising campaigns being multiplied risk of complications. Thus, the present study aimed to conduct a review of the risks and benefits related to calcium supplementation performed, usually without medical guidance in elderly.
\end{abstract}

Key Words: elderly, calcium, supplementation

\section{RESUMO}

O cálcio é um dos minerais de importância ímpar no organismo humano, exercendo importantes funções celulares, principalmente sobre o sistema ósseo. Com o envelhecimento, alguns órgãos sofrem alterações que comprometem o seu funcionamento, como no caso do osso que tem a sua densidade reduzida a partir do envelhecimento, aumentando o risco de fraturas e de doenças ósseas como, por exemplo, a osteoporose. Uma opção para diminuir este desgaste ósseo é a utilização de suplementos medicamentosos à base de sais de cálcio, os quais possuem concentrações diferentes de cálcio, além de necessitarem de um $\mathrm{pH}$ estomacal ótimo para absorção e apresentarem diferentes graus de solubilidade, podendo, portanto, serem utilizados em diferentes situações clínicas. Além da redução da perda óssea, tal forma de tratamento possui outros benefícios como a redução da pressão sanguínea e redução da dislipidemia, mas também está associado à calcificação vascular e outros riscos que promovem o aumento da mortalidade nos pacientes, além de promover o acúmulo de metais pesados. Como estes suplementos podem ser adquiridos sem prescrição médica, os mesmos estão facilmente ao alcance da população idosa que é induzida pelas campanhas publicitárias, sendo risco de ocorrência de complicações multiplicado. Com isso, o presente estudo teve por objetivo realizar uma revisão sobre os riscos e benefícios relacionados à suplementação medicamentosa de cálcio realizada, geralmente sem orientação médica, por idosos.

Palavras-Chave: idosos, cálcio, suplementação, 


\section{INTRODUÇÃO}

O envelhecimento humano é um conjunto de alterações fisiológicas que reduz a capacidade de adaptação do indivíduo ao meio ambiente. Trata-se de um processo biológico, estocástico e universal, que ocorre de forma dinâmica e progressiva, mas que pode afetar a integridade do indivíduo e permitir que doenças crônicas desenvolvam-se, o que causa um impacto grande sobre a saúde e a qualidade de vida das pessoas $(1,2)$.

Com a senilidade, diversos sistemas são comprometidos, principalmente cardíaco, respiratório, musculoesquelético, nervoso, gastrintestinal e urinário $(3,4)$ No sistema musculoesquelético podem surgir enfermidades crônicas como as doenças osteoarticulares; no gastrintestinal, as alterações referem-se à redução no fluxo sanguíneo, nas secreções ácidas e de pepsina, na superfície de absorção, no peristaltismo e nas secreções de enzimas pancreáticas e hepáticas, que acabam por comprometer a absorção de nutrientes e de medicamentos. Já no sistema urinário ocorre principalmente à diminuição da função renal devido à redução do fluxo sanguíneo renal e da filtração glomerular, o que, no caso dos fármacos de excreção renal, pode gerar acúmulos e, consequentemente, toxicidade $(2,5)$.

Independentemente do grau de comprometimento, algumas das alterações funcionais são notadas durante a realização das atividades cotidianas, como no caso daquelas em que se faz uso do sistema ósseo. O osso é um órgão formado por cálcio, que é um mineral imprescindível às funções biológicas. Cerca de $99 \%$ do cálcio do organismo encontram-se sob a forma de hidroxiapatita, que promove o suporte estrutural do esqueleto. O restante está distribuído entre os dentes e os tecidos moles, sendo que apenas $0,1 \%$ encontra-se no líquido extracelular $(6,7)$. Contudo, a principal função biológica exercida pelo cálcio orgânico é a de atuar como segundo mensageiro em respostas a sinais extracelulares, além de atuar na contração muscular, na coagulação sanguínea, no metabolismo celular, na transmissão do impulso nervoso e como co-fator enzimático (7-13).

Esse trabalho de revisão da literatura tem por objetivo descrever os riscos e benefícios relacionados à suplementação medicamentosa de cálcio realizada, geralmente sem orientação médica, por idosos.

\section{Homeostase do cálcio}

Para que as funções metabólicas sejam realizadas, o nível sérico de cálcio deve ser mantido em torno de 8,8 a $10,6 \mathrm{mg} / \mathrm{dL}$ (14). Esse nível é obtido através de um processo dinâmico que envolve diversos órgãos, sendo a glândula paratireoide o principal centro para a manutenção desse mineral, pois ela, juntamente com as células $\mathrm{C}$ secretoras de calcitonina da glândula tireoide, percebe desvios sutis na concentração plasmática de cálcio. Além disso, para conservação desse balanço, uma íntima relação deve existir entre a ingestão, a excreção e a absorção, sendo que esta última refere-se à diferença entre a ingestão e a perda fecal $(6,12,15)$. Como esse mineral não é produzido pelo organismo, o mesmo deve ser adquirido através da ingestão diária de alimentos que o contém, como os alimentos lácteos de baixo teor de gordura, alimentos enriquecidos com cálcio ou através da administração de suplementos farmacológicos (911,13).

A absorção de cálcio está intrinsecamente relacionada com a capacidade absortiva do intestino, a quantidade do mineral ingerida, a faixa etária e as condições clínicas do indivíduo, de modo que quando a oferta é pequena, a absorção torna-se aumentada e vice-versa. Desta forma, quanto maior a necessidade do organismo e menor a oferta desse mineral, mais eficiente será a absorção $(13,16)$.

Assim, o cálcio ingerido mistura-se com o cálcio endógeno, o qual foi secretado no suco digestivo, e ambos são absorvidos no intestino através de dois mecanismos: o transporte ativo e a difusão passiva $(6,8,14$, 17). Se há uma baixa oferta de cálcio na dieta, este é absorvido ativamente em todo o intestino delgado, principalmente no duodeno e no jejuno proximal, devido ao pH ser mais ácido, pois o cálcio precipita-se em meio alcalino, e devido à presença de uma proteína ligadora de cálcio, a calbidina. Esse mecanismo é saturável e dependente da presença de calcitriol (1,25-dihidroxivitamina D), que é um metabólito ativo da vitamina D. Entretanto, se há uma alta ingestão de cálcio, superior a $500 \mathrm{mg} / \mathrm{dia}$, o mecanismo de absorção por transporte ativo satura-se e o cálcio passa a ser absorvido por difusão passiva, em um processo de absorção entre as células, denominado de paracelular, na porção do jejuno distal e do íleo $(6,8$, $13,14,18)$.

Do total de cálcio ingerido na dieta, apenas 30 a $40 \%$ é absorvido pelas células do intestino, sendo que hormônios como o da paratireoide (PTH), do crescimento, estrogênio e a progesterona podem aumentar, por diferentes mecanismos, a quantidade absorvida deste mineral $(6,11,12,14,17,19)$.

O cálcio que é absorvido por transporte ativo é deslocado para o interior de organelas como mitocôndrias e retículo endoplasmático onde é estabilizado sob formas não cristalinas até ser liberado para a corrente sanguínea (13). Na corrente sanguínea, este mineral é carregado de três formas diferentes: de 45 a $50 \%$ ligado a proteínas, globulina, prealbumina e, principalmente, albumina; $8 \%$ complexado com sulfato, fosfato e citrato; e $50 \%$ na forma ionizada, que é a porção funcional (13, 
20). Estas formas de transporte na corrente sanguínea interferem na filtração renal do cálcio, pois o cálcio ligado à albumina não é ultrafiltrável, mas o cálcio complexado e o iônico são $(8,13,20)$.

A concentração sanguínea de cálcio é controlada pelo calcitrol, hormônio paratireoidiano e calcitonina. Além disso, o metabolismo mineral ósseo influencia a concentração de íons cálcio ao absorver ou libertar esse íon no sangue, que o transporta aos mais diversos locais para poder exercer suas funções orgânicas, sendo, inclusive armazenado no osso e nas paredes internas das artérias $(8,13,20-22)$.

Quando a concentração sanguínea de cálcio sutilmente declina a níveis abaixo do normal, a glândula paratireoide imediatamente libera o PTH para provocar aumento da concentração sérica de cálcio $(12,23,24)$. Para tal, esse hormônio atua de três formas diferentes, assim, ele age diretamente sobre os ossos e os rins e indiretamente sobre o intestino $(23,24)$. No sistema ósseo, o PTH aumenta a taxa de modificação do osso e de remodelagem óssea por acionar a atividade dos osteoclastos que promovem a mobilização do cálcio e fósforo em direção à corrente sanguínea; nos rins, o PTH promove um aumento da reabsorção de cálcio nos túbulos renais enquanto aumenta a excreção de fósforo; e no intestino ocorre otimização da absorção do cálcio e do fósforo dietético devido à estimulação da síntese de calcitrol nos rins $(23,24)$.

Dependendo da dieta e realização de atividade física, também há uma considerável perda de cálcio através do cabelo, suor, unhas, pele, fezes, urina e secreções digestivas (11). A porção excretada nas fezes refere-se ao cálcio da dieta que não foi absorvido e ao cálcio secretado no suco digestivo que não foi reabsorvido $(6,11$, $12,18,25)$.

A perda urinária de cálcio, em torno de 140 a 200 $\mathrm{mg} /$ dia, refere-se ao cálcio que é filtrado nos rins, mas que não é reabsorvido pelos túbulos renais $(6,19)$. Essa perda decorre quando o cálcio ionizado e o cálcio complexado presentes na corrente sanguínea são filtrados no glomérulo passando a compor parte do filtrado glomerular que percorrerá os túbulos renais para então formar a urina. No túbulo proximal são reabsorvido cerca de $50 \mathrm{a}$ $60 \%$ de cálcio através do método paracelular e, na porção arce dente da alça de Henle, também pelo método passivo, aproximadamente, 30 a $35 \%$. E no túbulo contornado distal, finalmente, $10 \%$ do cálcio filtrado é reabsorvido transcelularmente. Isso totaliza uma reabsorção de 90 a $95 \%$ de todo o cálcio filtrado no glomérulo $(6,19,20)$.

O cálcio perdido pela pele, unhas e cabelo é considerado como insignificante, pois compreende uma perda de 40 a $80 \mathrm{mg}$ de cálcio, sendo considerado irrelevante frente à ingesta de cálcio $(6,19)$. Assim, em indivíduos normais, a quantidade de cálcio que é perdida pelas fezes e pelos rins é igual àquela que é absorvida da dieta (25).

\section{Homeostase do cálcio no idoso}

Para que os níveis adequados de cálcio sejam mantidos, a recomendação de ingestão diária (RID) varia de 600 a $1200 \mathrm{mg}$, dependendo da necessidade do cálcio, a qual varia de pessoa para pessoa, entre os sexos, de região para região e conforme a faixa etária, sendo maior em períodos em que a necessidade orgânica de cálcio é maior, como os períodos de crescimento e na realização de atividades físicas que exijam alta densidade óssea ( 9 , $14,16,18)$. Já na vida adulta, a demanda de cálcio é reduzida, salvo condições específicas, como gravidez, lactação e senilidade $(11,18)$.

A partir dos 35 anos, o processo de depleção da massa óssea se inicia, pois há prevalência do processo de reabsorção de cálcio devido à ingesta deficiente de nutrientes, à exposição solar reduzida e à diminuição da absorção de cálcio no intestino $(9,14,26)$. Entretanto, nas mulheres, devido ao climatério, o nível de estrogênios diminui e faz com que a perda óssea ocorra mais aceleradamente (14).

Em alguns indivíduos com mais de 60 anos pode ocorrer a diminuição da secreção de ácido clorídrico pelo estômago, o que compromete a absorção do cálcio ingerido. Somado a isso, têm-se uma deficiência na absorção intestinal, na metabolização renal e hepática e na síntese de vitamina $\mathrm{D}(9,13)$. Além disso, com 75 anos ou mais, a taxa de filtração glomerular reduz-se à metade, pois $30 \%$ dos glomérulos não estão funcionais, devido à esclerose. Desta forma, compromete-se o processo de reabsorção de cálcio nos túbulos proximais (20).

Em alguns casos, paralelamente ao envelhecimento, há o desenvolvimento de doenças crônicas renais, que são desencadeadas principalmente devido à diabetes e hipertensão. Nesses casos, a gravidade da doença desencadeia significativas alterações na homeostase do cálcio, pois pode provocar uma incapacidade renal na reabsorção tubular e uma irregularidade em sua absorção intestinal (20).

Todas estas consequências do envelhecimento causam uma redução na concentração sérica do cálcio, pois promovem uma aumentada produção e liberação de PTH para reverter o quadro (23). Como este gerencia a reabsorção do cálcio ósseo, no envelhecimento há uma perda de cálcio maior do que sua absorção/reabsorção, portanto há um aumento da perda óssea, o que pode propiciar o surgimento de patologias ósseas, como a osteoporose $(11,16,18,23)$. 


\section{MATERIAL E MÉTODOS}

Este estudo foi realizado através de buscas bibliográficas em revistas, teses e periódicos científicos nacionais e internacionais, encontrados nas bases de buscas bibliográficas como Scielo, PubMed, Google acadêmico, BIREME, MEDLINE e LILACS. As buscas foram realizadas por meio da utilização das palavras chaves: suplementação de cálcio, "calcium suplementation", suplementação de cálcio nos idoso, toxicidade do cálcio, benefícios da suplementação de cálcio, "calcium toxicity", "Elderly and the use of calcium".

Para a seleção da bibliografia, os critérios utilizados foram a abordagem da suplementação por cálcio nos idosos, os benefícios que esta suplementação acarreta e os riscos que a mesma pode desencadear e os casos na qual ela é necessária. Foram excluídas da pesquisa, aquelas bibliografias que não atenderam a temática da pesquisa.

\section{RESULTADOS E DISCUSSÃO}

\section{Suplementos medicamentosos de cálcio}

Nos casos em que a ingesta diária não alcança os níveis recomendados, opta-se pela ingestão acessória de suplementos farmacológicos de cálcio para suprir o déficit deste íon na alimentação $(9-11,13)$. Assim, geralmente esses suplementos devem ser utilizados pelos indivíduos que eliminaram ou restringiram da dieta leite e seus derivados, na alergia alimentar, naqueles com doenças relacionadas com o metabolismo ósseo, entre outros (9).
Estão disponíveis para suplementação os sais de cálcio sob a forma de acetato, carbonato, citrato, citrato malato, cloreto, fosfato dibásico, fosfato tricálcico, glubionato, gliconato e lactato. Estes sais diferem-se entre vários aspectos como o perfil de solubilidade apresentado, a quantidade de cálcio, o valor do $\mathrm{pH}$ ideal para absorção, devendo ser utilizados em diferentes casos clínicos (Tabela 1) $(9,13,27)$.

Apesar de todas as formulações mostrarem $\mathrm{O}$ citrato e o carbonato são os principais sais de cálcio, pois os demais sais possuem uma quantidade de cálcio elementar muito reduzida e seria preciso uma múltipla administração diária para atingir níveis séricos fisiologicamente adequados $(27,28)$. Sabe-se que a baixa disponibilidade do cálcio também é uniformemente observada em todos esses suplementos de sais de cálcio (19). E embora a solubilidade dos sais não cause interferência na absorção dos mesmos, as propriedades relacionadas interferem na eficácia do tratamento, pois todos os sais necessitam de condições adequadas para serem absorvidos e estarem biodisponíveis.

Nesta concepção, para ser bem absorvido, o carbonato de cálcio necessita de um $\mathrm{pH}$ mais baixo, por isso deve ser ingerido durante as refeições, o que torna o citrato o sal de cálcio mais indicado em casos com $\mathrm{pH}$ estomacal é elevado, pois nesta situação esse sal poderá ser mais absorvido $(9,13)$. Devido a isso, o carbonato não deve ser utilizado como suplemento por pessoas que possuem gastrite atrófica ou qualquer outra patologia que aumente o pH gástrico, e o citrato, sal mais solúvel e absorvido em $\mathrm{pH}$ menos ácido, é o suplemento adequado para casos de pacientes com hipocloridria (13).

Tabela 1 - Propriedades gerais dos sais de cálcio

\begin{tabular}{|c|c|c|c|c|}
\hline Sais de cálcio & $\begin{array}{c}\text { \% cálcio } \\
\text { elementar }\end{array}$ & $\begin{array}{c}\text { Cálcio elementar } \\
(\mathbf{m g} / \mathbf{g})\end{array}$ & Solubilidade em água & $\begin{array}{c}\text { Parâmetros para } \\
\text { solubilidade em água }\end{array}$ \\
\hline Acetato & 25,3 & 253 & Solúvel & $34.7 \mathrm{~g} / 100 \mathrm{~mL}\left(20^{\circ} \mathrm{C}\right)$ \\
\hline Carbonato & 40 & 400 & Pouco solúvel & $0,014 \mathrm{~g} / 100 \mathrm{~mL}\left(20^{\circ} \mathrm{C}\right)$ \\
\hline Citrato & 21,1 & 211 & Solúvel & $0.095 \mathrm{~g} / 100 \mathrm{~mL}\left(25^{\circ} \mathrm{C}\right)$ \\
\hline Citrato malato & 13 & 130 & Solúvel & * \\
\hline Cloreto de cálcio & 27,2 & 272 & Solúvel & $74.5 \mathrm{~g} / 100 \mathrm{~mL}\left(20^{\circ} \mathrm{C}\right)$ \\
\hline Fosfato dibásico & 24,4 & 230 & Pouco solúvel & $*$ \\
\hline Fosfato tricálcico & 38,8 & 380 & Pouco solúvel & $*$ \\
\hline Glubionato & 6,5 & 65 & Solúvel & $*$ \\
\hline Gliconato & 9,3 & 93 & Solúvel & $*$ \\
\hline Lactato & 13 & 130 & Solúvel & $*$ \\
\hline
\end{tabular}

* Dados não encontrados 


\section{Benefícios da suplementação de cálcio nos idosos}

A importância desta suplementação reside nos benefícios por ela apresentada, como a diminuição da perda da massa óssea, o aumento da sua densidade, redução do colesterol total e frações, redução da pressão sanguínea, dentre outras $(9,21,22,29)$.

O osso é a reserva natural de cálcio no organismo, e é dele que é retirado o cálcio para suprir a necessidade orgânica naqueles casos em que há baixa absorção de cálcio ou quando a excreção suplanta a absorção. Com a administração dos suplementos, eleva-se a quantidade sanguínea de cálcio de modo a impedir a retirada do cálcio ósseo, pois impede a retirada do cálcio do osso e aumenta sua absorção, portanto, aumenta a densidade e diminui a degradação da massa óssea. Além disso, essa alta ingesta reduz a excessiva remodelagem que geralmente ocorre em mulheres pósmenopausadas e em osteoporóticos, sendo que a remodelagem é um fator de risco para a fratura (7). Independente de qual seja o tipo de suplementação prescrita, o efeito de redução do risco de fraturas e aumento da densidade óssea somente ocorre se houver adesão ao tratamento (30).

Um inesperado benefício da suplementação de cálcio, principalmente em mulheres, é a diminuição da concentração sérica de lipídios (31). Isso ocorre devido à ligação do cálcio ingerido com ácidos biliares e ácidos graxos no intestino, ocasionando na redução da absorção da gordura $(22,31)$. Além disso, com o uso da suplementação, o nível sérico de cálcio mantém-se entre os parâmetros da normalidade e, portanto, reduz-se a concentração de PTH circulante, havendo uma baixa necessidade de calcitrol, pois a alta oferta de cálcio favorece a absorção do tipo paracelular, o qual não faz uso dessa vitamina $(22,31)$. Com uma reduzida quantidade de PTH e/ou de vitamina D, existe uma menor quantidade de cálcio intracelular, promovendo-se a redução do fluxo de cálcio nos adipócitos, desencadeando a lipólise e inibindo a lipogênese, e, assim, propicia efeitos favoráveis nos lipídios e nas lipoproteínas, como o colesterol e as suas frações $(28,31)$. Esse mesmo mecanismo somado com o aumento da oxidação da gordura nos adipócitos também desencadeia a perda de peso, de modo a se observar uma variação de $3 \%$ no peso corporal $(22,29)$.

O efeito natriurético que o cálcio promove e a redução nos níveis de PTH e calcitrol e, consequentemente, nos níveis de concentração intracelular de cálcio, desencadeiam uma reversão do efeito pressor promovido pelo cálcio nas células do músculo liso e uma diminuição na resistência vascular periférica, e, dessa forma, observa-se uma redução no risco de hipertensão, pois há uma pequena mas significativa redução na pressão sistólica e diastólica (22, 29, 31, 32). Além disso, a suplementação de cálcio pode elevar a concentração de vasodilatadores que podem agir mais intensamente no sistema renina-angiotensina-aldosterona. Entretanto, em alguns casos, ocorre apenas redução na pressão sistólica (29).

\section{Riscos da suplementação de cálcio nos idosos}

Como citado anteriormente, o organismo dos idosos não funciona da mesma forma que o organismo de um indivíduo adulto, assim, alguns riscos referentes à suplementação estão associados a uma maior ocorrência neste grupo etário. Alguns desses riscos são a calcificação vascular, o aumento no risco de infarto no miocárdio, o aumento da mortalidade em paciente renais, cálculo renal, câncer, entre outros $(9,21,22,29)$.

Nas paredes dos vasos sanguíneos são expressos receptores sensíveis ao cálcio, sendo que a calcificação arterial é regulada por vários inibidores, os quais incluem o pirofosfato. Quando há aumento da concentração sérica de cálcio, é esperado que este completa com o pirofosfato, provocando a redução da capacidade de inibição da calcificação vascular $(22,30)$. Além disso, quando há suplementação, o cálcio é rapidamente retido nas artérias e lentamente nos ossos, o que contribui para o aumento da calcificação (22).

No caso de indivíduos com arteriosclerose, a calcificação vascular é tida como um marcador subclínico, pois há um mecanismo de deposição de cálcio nas paredes dessas artérias coronárias e aorta e, assim, há espaçamento da placa aterosclerótica $(30,31)$. Além disso, a calcificação vascular é um dado informativo prévio de doença vascular, como a doença cardíaca coronariana e doença cardiovascular (33).

Outro risco relacionado à calcificação do músculo liso vascular tem sido a ocorrência de lesões cerebrais, as quais são marcadores de doença cerebrovascular, além de que essas lesões também estarem ligadas ao aumento do risco de depressão, disfunção cognitiva e demência (31).

Uma das funções do cálcio é agir como um co-fator na coagulação sanguínea e sua ingestão suplementar pode estar associada a pequenas alterações na coagulação. Além disso, os receptores cálcio dependentes existentes nas plaquetas, fazem com que elas respondam diretamente a mudanças na concentração sanguínea do mineral. Desta forma, propicia-se a o aparecimento do coágulo, o que aumenta o risco de infarto, pois a formação de coágulos nos vasos do coração é um passo crítico para ocorrência dessa enfermidade (30).

Esses riscos comumente agravam-se em pacientes com falha renal, em diálise ou em pré-diálise, porque a excreção urinária de cálcio está reduzida $(30,33)$. Nesses pacientes, a ocorrência da calcificação das principais artérias ocorre mais rapidamente do que nos idosos com rins funcionando normalmente, além de que é uma alta 
causa de mortalidade no pacientes com falha renal (22). Nesses casos observa-se também a ocorrência de acidente vascular encefálico (AVE) e, nos casos em que há associação do cálcio com o fosfato pode haver risco de morte (20).

Cálculos nos rins ou litíase renal são causadas principalmente pela excreção de cálcio e oxalato, assim, se há elevação da absorção de cálcio ou oxalato, há aumento de sua excreção e, consequentemente, aumento do risco de ocorrência de litíase, sendo o oxalato um fator de risco para a ocorrência de litíase maior do que o cálcio (7). Aproximadamente $80 \%$ dos cálculos contém cálcio em sua composição e o risco de desenvolvê-las é aumentado em pessoas que são predispostas para esse tipo de patologia e é depende, também, da fonte de cálcio. Contudo, uma suplementação de 200 a $500 \mathrm{mg} / \mathrm{dia}$ de cálcio relaciona-se com o aumento da ocorrência dessas pedras, o que não ocorre quando há aumento do cálcio dietético, pois com o aumento desse tipo de cálcio, a absorção de oxalato é reduzida, o que, consequentemente, diminui o risco de surgimento dessas pedras $(7,31,34)$.

Embora tido como um dos benefícios da suplementação de cálcio, a complexação deste com ácidos graxos e biliares faz com que estes não sejam absorvidos, causando um efeito irritativo na mucosa intestinal, o que serve como promotor para o desenvolvimento do câncer de colon, embora este seja um mecanismo altamente complexo (7).

Alguns suplementos podem conter em sua composição metais pesados como chumbo, o qual tem um limite permitido de 7,5 microgramas a cada $1000 \mathrm{mi}-$ ligramas de cálcio. Entretanto, tais suplementos podem não ser testados antes de serem disponibilizados para venda, o que promove a intensificação da acumulação no organismo de metais pesados porventura presentes na formulação (28).

\section{CONCLUSÃO}

A análise de estudos referentes à suplementação medicamentosa de cálcio aponta que esses fármacos podem colaborar com a profilaxia das doenças ósseas desencadeadas pela senilidade. Entretanto, como são isentos de prescrição, muitos idosos fazem seu uso sem qualquer tipo de acompanhamento médico ou farmacêutico.

Os benefícios oriundos do aumento sérico de cálcio devido à suplementação são reais e proporcionam uma significativa melhora na execução das atividades diárias que os idosos realizam. Além de, em alguns casos, estar associada a outros benefícios como a redução da concentração sérica de lipídios. Entretanto, agregada a essa ingestão podem surgir alguns riscos, como a calcificação vascular que pode vir culminar em infarto ou causar algum problema neurológico. Estes riscos contrastam com os mínimos efeitos que os alimentos enriquecidos com cálcio promovem na concentração sérica de cálcio.

Além disso, a dose de suplementos que produzem efeitos benéficos nessa faixa etária ainda não é clara e há mínimos efeitos quando se faz ingestão de alimentos enriquecidos com esse mineral. Assim, deve-se observar o risco e o benefício da necessidade dessa suplementação.

\section{REFERÊNCIAS}

1. Silva WJM, Ferrari CKB. Metabolismo mitocondrial, radicais livres e envelhecimento. Rev. Bras. Geriatr. Gerontol., Rio de Janeiro, 2011; 14: 441-451.

2. Soares MAMS. Avaliação da terapêutica potencialmente inapropriada no doente geriátrico. [Tese de doutorado em farmacoepidemiologia]. Lisboa, Portugal: Universidade de Lisboa. 2009.

3. Santello FH, Redigolo E, Toniello WM da M, Monteiro SCM. Perfil da automedicação em idosos no Município de Barretos/São Paulo/ Brasil. Infarma. 2013; 25 (1): 32-26.

4. Fechine B, Trompieri N. O processo de envelhecimento: as principais alterações que acontecem com o idoso com o passar dos anos. Rev. Cienc. Int.. 2012; 20 (1): 106-194.

5. Cardoso AF. Particularidades dos idosos: uma revisão sobre a fisiologia do envelhecimento. Educación Física y Deportes, revista digital, Buenos Aires, 2009; 130:
6. FAO, Food and Agriculture Organization of the United Nations. Human vitamin and mineral requirements. Report of a joint FAO/WHO expert consultation Bangkok, Thailand. 2001 [14 set 2013]. Disponível em <ftp://ftp.fao.org/docrep/fao/004/y2809e/ y2809e11.pdf $>$

7. Heaney RP. Calcium intake and disease preventation. Arq. Bras. Endocrinol. Metab. 2006; 50 (4):

8. Chung M. Vitamin D and Calcium: A Systematic Review of Health Outcomes. Evidence Report No. 183. (Prepared by the Tufts Evidence-based Practice Center under Contract No. HHSA 2902007-10055-I.) AHRQ Publication No. 09-E015. Rockville, MD: Agency for Healthcare Research and Quality. Rockville, MD, 2009.

9. Pereira GAP, Genaro OS, Pinheiro MM, Szejnfeld VL, Martini LA. Cálcio dietético - estratégias para otimizar o consumo. Rev. Bras. Reumat. 2009; 49(2): 164-180. 
10. Bueno AL, Czepielewski MA. The importance for growth of dietary intake of calcium and vitamin D. Jornal de Pediatria, Rio de Janeiro, 2008; 84(5): 386-394.

11. Bedani R, Rossi EA. O consumo de cálcio e a osteoporose. Semina: Ciências Biológicas e da Saúde, Londrina, 2005; 26(1): 3-14.

12. Brandt M. Calcium homeostasis. 1999 [05 set. 2013]. Disponível em $<$ http://www.rosehulman.edu/ brandt/Chem330/EndocrineNotes/Chapter_8_Calcium.pdf $>$

13. Grüdtner VS, Weingrill P, Fernandes AL. Aspectos da absorção no metabolismo do cálcio e vitamina D. Rev. Bras. Reumat. 1997; 37(3):

14. Aquino AC, Oliveira LC, Wagner R. Dosagem sérica de cálcio em idosos de instituições de amparo. Cadernos da Escola de Saúde, Curitiba, 2010; 4(1): 138-148.

15. Kurokawa $\mathrm{K}$. How is plasma calcium held constant? Milieu interieur of calcium. Kidney International, 1996; 49: 1760-1764.

16. Buzzinaro EF, Almeida RNA de, Mazeto GMFS. Biodisponibilidade do cálcio dietético. Arq Bras Endocrinol Metab [online]. 2006, 50(5): 852-861.

17. NIH. Consensus Conference Development on Optimal Calcium Intake. Optimal calcium intake. JAMA, 1994; 272(24): 1942-1948.

18. Barbosa CR, Andreazzi MA. Intolerância à lactose e suas consequências no metabolismo do cálcio. Revista Saúde e Pesquisa, 2011, 4(1): p. 81-86.

19. Bhatia V. Dietary calcium intake - a critical reappraisal. Indian Journal Medicina Residence, 2008; 127: 269-273.

20. Tejwani V, Qian Q. Calcium regulation and bone mineral metabolism in elderly patients with chronic kidney disease. Nutrients, 2013; 5: 1913-1936.

21. Bolland MJ, Avenell A, Baron JA, Grey A, Maclennan GS, Gamble GD, Reid IR. Effect of calcium supplements on risk of myocardial infarction and cardiovascular events: meta-analysis. BMJ on line, 2010; 341

22. Reid IR, Bolland MJ, Grey A. Does calcium supplementation increase cardiovascular risk? Clin. Endocrinol., 2010; 73: 689-695.
23. Vescini F, Grimaldi F. PTH 1-84: bone rebuilding as a target for the therapy of severe osteoporosis. Clinical Cases in Mineral and Bone Metabolism, 2012; 9(1): 31-36.

24. Freitas LCC de. Dinâmica da secreção de paratormônio após paratireoidectomia total e autotransplante. Ribeirão Preto, 2007. 130 p.

25. Nordin BEC. Calcium in health and disease. Food, nutrition and agriculture. 1997.

26. Austrália. Calcium medical guide. 2012 [21 set. 2013]. Disponível em <http://www.arthritis.org.au/content/Document/oa_medical_calcium.pdf $>$

27. Hardman JG, Limbird LE. Goodman \& Gilman: As Bases Farmacológicas da Terapêutica. McGraw Hill, 11 ${ }^{\mathrm{a}}$ ed. 2010.

28. Houtkooper L, Farrell VA. Calcium Supplement Guidelines. Tucson, AZ: University of Arizona Cooperative Extension. 2011 [19 set. 2013]. Disponível em < http://ag.arizona.edu/pubs/health/ az1042.pdf $>$

29. Reid IR, Horne A, Mason B, Ames R, Bava U, Gamble G D. Effects of calcium supplementation on body weight and blood pressure in normal older women: a randomized controlled trial. J. Clin. Endocrinol. Metab., 2005; 90(7): 3824-3829.

30. Reid IR, Bolland MJ, Sambrook PN, Grey A. Calcium suplementation: balancing the cardiovascular risks. Maturitas, 2011; 69: 289- 295.

31. Daly RM, Ebeling PR. Is Excess Calcium Harmful to Health?. Nutrients, 2010; 2; 505-522.

32. Zemel MB. Calcium modulation of hypertension and obesity: mechanisms and implications. J. Am. Coll. Nutrition. 2001; 20(5); 428S-435S.

33. Spence LA, Weaver CM. Calcium intake, vascular calcification, and vascular disease. Nutrition Reviews, 2013; 71(1): 15-22.

34. Austrália. Calcium consumer guide. 2012 [20 out. 2013]. Disponível em <http://www.arthritis.org.au/content/Document/oa_consumer_calcium.pdf $>$ 